\title{
Aptidão funcional de idosas praticantes de atividades físicas
}

\author{
Functional fitness of older women performing physical activities
}

1. Universidade Federal de Santa Catarina, Centro de Desportos, Núcleo de Pesquisa em Cineantropometria e Desempenho Humano. Florianópolis, SC. Brasil.

2. Universidade Federal de Santa Catarina, Centro de Desportos, Florianópolis, SC. Brasil.

Recebido em 05/09/09 Revisado em 16/10/09 Aprovado em 24/11/09
Resumo - O envelhecimento é um processo progressivo e natural e a atividade física vem sendo apontada como uma possibilidade de minimizar o seu impacto e, especialmente, manter a capacidade funcional do idoso por mais tempo. O objetivo do presente estudo foi analisar as modificações do Índice de Aptidão Funcional Geral (IAFG) de idosos participantes de um programa de atividades físicas no decorrer de 10 meses, associando aos diferentes estratos etários, tempo de participação no programa e modificações nas capacidades físicas avaliadas. O estudo foi realizado com 225 idosos, média de idade de 69,26 anos (dp=5,685), participantes do programa de Atividade Física e Dança Folclórica para a Terceira Idade do Centro de Desportes/Universidade Federal de Santa Catarina. Para a análise dos dados foi utilizada a estatística descritiva, o teste $t$ de Student e análise de variância (ANOVA). De março a dezembro de 2005, verificou-se diferença estatisticamente significante $(p<0,01)$ do IAFG e nas variáveis agilidade/equilíbrio e coordenação $(\mathrm{p}<0,01)$. As capacidades físicas força $(p=0,323)$, resistência $(p=0,946)$ e flexibilidade $(p=0,722)$ não tiveram diferença significante no período analisado. Verificou-se também que o grupo que praticava atividades físicas há mais de 10 anos e com faixa etária acima de 80 anos de idade, apresentaram melhor IAFG nas avaliações realizadas. A prática de atividades físicas por idosos pode ser um importante fator para a melhora e/ou manutenção da aptidão funcional no decorrer do processo de envelhecimento.

Palavras-chave: Aptidão funcional; Envelhecimento; Atividade física.

Abstract - Aging is a natural and progressive process and physical activities have been indicated as a possibility to minimize its impact and, especially, to maintain the functional capacity of older adults for a longer period of time. The objective of the present study was to analyze modifications in the General Functional Fitness Index (GFFI) in older adults participating in a physical activity program for a period of 10 months and to associate the results with different age strata, duration of participation in the program and modifications in physical fitness. The study was conducted on 225 older women (mean age: 69.26, sd=5.685), participating in the Physical Activity and Folk Dance Program for The Elderly of the Sports Center, Universidade Federal de Santa Catarina. Descriptive statistics, Student t-test and analysis of variance (ANOVA) were used for analysis of the data. A significant difference $(p<0.01)$ in GFFI and in the variables agility/balance and coordination $(p<0.01)$ was observed between March and December 2005. Strength $(p=0.323)$, resistance $(p=0,946)$ and flexibility $(p=0.722)$ did not differ significantly during the period analyzed. In addition, the group performing physical activity for more than 10 years and the group older than 80 years presented the best GFFI in the assessments. Physical activity for older adults might be an important factor to improve and/or maintain functional fitness during the process of aging.

Key words: Functional fitness; Aging; Physical activity. 


\section{INTRODUÇÃO}

O envelhecimento é um processo progressivo, contínuo, natural e irreversível, que provoca desgastes e alterações em vários sistemas funcionais e diferenciam-se de um indivíduo para o outro1.

A capacidade funcional, especialmente no que se refere à dimensão física, é um dos importantes marcadores de um envelhecimento bem sucedido e de uma melhor qualidade de vida. Assim, a manutenção e a preservação da capacidade física ou funcional para desempenhar as atividades básicas da vida diária é um ponto essencial para prolongar, por maior tempo possível, a independência, oportunizando uma vida mais saudável ${ }^{2}$.

Gobbi $^{2}$ destaca que uma das principais formas de evitar, minimizar e/ou reverter a maioria dos declínios físicos, sociais e psicológicos que, frequentemente, acompanham o idoso, é a atividade física, demonstrando que ela está constantemente associada a melhoras significativas nas condições de saúde, como o controle do estresse, da obesidade, do diabetes, das doenças coronarianas e, principalmente, a melhora da aptidão funcional do idoso ${ }^{3}$.

Dessa forma, a participação em programas de atividade física, em que os idosos realizam trabalho de força, de flexibilidade, de agilidade, de resistência aeróbia e de coordenação é fundamental para realizar as suas tarefas diárias, minimizando o risco de desenvolver doenças que podem levar à dependência4.

A relação aptidão funcional e atividade física vem sendo explorada em pesquisas. Todavia, ainda existem lacunas na literatura sobre as diferenças da aptidão funcional em função do estrato etário, do tempo de prática e da interferência da ginástica sobre as capacidades físicas.

Diante desse contexto, o objetivo principal deste trabalho foi analisar as modificações do Índice de Aptidão Funcional Geral (IAFG) de idosos participantes de um programa de atividades físicas, no decorrer de 10 meses. Também foram analisadas as modificações nas capacidades físicas, a interferência do tempo de participação no programa sobre o IAFG e a sua comparação com os diferentes estratos etários.

\section{PROCEDIMENTOS METODOLÓGICOS}

O estudo caracterizou-se como sendo de caráter experimental com delineamento pré-experimental - avaliações pré e pós-teste ${ }^{5}$, com intervenção de 10 meses.

A pesquisa obteve aprovação do Comitê de Ética para Seres Humanos da Universidade Federal de Santa Catarina (UFSC), processo nº 050/05, e os idosos assinaram o Termo de Consentimento Livre e Esclarecido - TCLE.

\section{Sujeitos}

Este estudo teve como população 474 idosos participantes do programa de Atividade Física e Dança Folclórica para a Terceira Idade, do Centro de Desportos/Universidade Federal de Santa Catarina (CDS/UFSC).

Os critérios de seleção da amostra foram: idosos do sexo feminino com idade igual ou superior a 60 anos; praticante de exercício físico; ter realizado todos os testes da bateria American Alliance for Health, Physical Education, Recreation and Dance (AAHPERD) nas avaliações de março e dezembro de 2005. Atenderam aos critérios acima 225 idosos, que constituíram a amostra desse estudo.

Os idosos participantes praticavam, pelo menos, duas sessões semanais de aulas de ginástica oferecidas pelo programa, com duração de cinquenta minutos cada sessão.

\section{Protocolo de avaliação e procedimentos}

Para avaliar a capacidade funcional, foi utilizada a bateria de testes para idoso desenvolvida pela AAHPERD ${ }^{6,7}$. Esta bateria é composta por cinco testes motores (flexibilidade, coordenação, agilidade/ equilíbrio dinâmico, resistência de força de membros superiores e resistência aeróbia geral) ${ }^{7}$, aplicados nesta sequência. A bateria da AAHPERD é uma bateria que atende a critérios de validade e confiabilidade ${ }^{8}$.

O Índice de Aptidão Funcional Geral (IAFG) é obtido pelo somatório dos índices percentis dos 5 testes.

Utilizaram-se os dados de identificação dos idosos registrados no Software CFI (Capacidade Funcional dos Idosos), no qual constam nome, idade, escolaridade, sexo, estado civil, tempo no programa, entre outras informações, que são coletadas quando estes iniciam as atividades físicas no programa e atualizadas anualmente.

A coleta dos dados da AAHPERD foi realizada em março e dezembro de 2005, nas dependências da UFSC/CDS, por uma equipe treinada, composta por acadêmicos e profissionais de Educação Física da UFSC.

\section{Tratamento Estatístico}

Utilizou-se a estatística descritiva com as medidas de tendência central, dispersão, frequência e tabelas de contingência. $O$ teste $t$ pareado de Student veri- 
ficou as diferenças entre as avaliações dos testes e do IAFG, e a análise de variância (ANOVA) para identificar as diferenças em relação ao tempo de participação no programa e estratos etários (60-69; 70-79; 80 ou mais). O nível de significância adotado foi de $5 \%$.

\section{RESULTADOS}

Na Tabela 1, podem ser visualizadas as características sociodemográficas dos idosos participantes do estudo como idade, escolaridade, estado civil e tempo que participam do programa de atividades físicas do CDS/UFSC.

Tabela 1. Características sociodemográficas dos idosos participantes do programa de atividade física do CDS/UFSC no ano de $2005(\mathrm{n}=225)$.

\begin{tabular}{lcc}
\hline & Freqüência & $\%$ \\
\hline Idade & & \\
\hline $60-69$ anos & 125 & 55,5 \\
\hline $70-79$ anos & 11 & 39,6 \\
\hline 80 anos ou mais & & 4,9 \\
\hline Escolaridade & 19 & \\
Analfabeto & 136 & 60,4 \\
\hline Fundamental & 56 & 24,9 \\
\hline Médio & 14 & 6,2 \\
\hline Superior & & \\
\hline Estado civil & 88 & 39,1 \\
\hline Casado & 48 & 21,3 \\
\hline Viúvo & 89 & 39,6 \\
\hline Separado, solteiro, divorciado & & \\
\hline Tempo no programa & 52 & 23,1 \\
\hline Até 3 anos de prática & 82 & 36,4 \\
\hline 3 a 10 anos de prática & 91 & 40,4 \\
\hline Acima de 10 anos de prática & &
\end{tabular}

Observa-se que a maioria $(55,5 \%)$ dos participantes do estudo são idosos jovens, têm escolaridade até o ensino fundamental (68,8\%) e 40,4\% participam do programa há mais de 10 anos. Em relação ao estado civil, os percentuais são semelhantes entre aqueles que são separados, solteiros ou divorciados $(39,6 \%)$ e aqueles casados $(39,1 \%)$.

$\mathrm{Na}$ Tabela 2, podem ser visualizados os resultados da avaliação das capacidades físicas que compõem o IAFG nas duas coletas realizadas.

De acordo com os resultados da Tabela 2, identifica-se que a coordenação e a agilidade/equilíbrio dinâmico foram as capacidades físicas que sofreram alterações significativas $(p<0,01)$ no decorrer de 10 meses. Já as capacidades resistência de força de membros superiores $(p=0,323)$, resistência aeróbia geral $(p=0,946)$ e flexibilidade $(p=0,722)$ não tiveram diferença significante no período analisado. Porém, ao observar as médias das referidas capacidades, percebe-se que houve manutenção, o que se mostra importante no decorrer do processo do envelhecimento.

Na Tabela 3, estão apresentados os resultados do IAFG de março e dezembro de 2005. Os resultados demonstram uma diferença estatisticamente significante $(p<0,01)$, resultante da melhora no desempenho dos testes.

Tabela 2. Médias das capacidades físicas da bateria AAHPERD dos participantes do programa de atividade física entre os meses de março e dezembro de 2005 ( $n=225)$.

\begin{tabular}{lccccc}
\hline $\begin{array}{l}\text { Média } \\
2005\end{array}$ & FLEX & COO & AGIL & RESISFOR & RAG \\
\hline Março & 60,95 & 12,89 & 26,50 & 20,94 & 542,82 \\
Dezembro & 60,74 & 11,32 & 25,04 & 21,20 & 543,15 \\
$t$ Student & 0,722 & $<0,01^{*}$ & $<0,01^{*}$ & 0,323 & 0,946 \\
\hline
\end{tabular}

$* \mathrm{p}<0,05$. Legenda: FLEX = Flexibilidade; $\mathrm{COO}=$ Coordenação; AGIL = Agilidade/Equilíbrio Dinâmico; RESISFOR = Resistência de Força dos membros superiores; RAG = Resistência Aeróbia Geral.

Tabela 3. Classificação do Índice de Aptidão Funcional Geral - IAFG dos participantes do programa de atividade física entre os meses de março e dezembro de $2005(n=225)$.

\begin{tabular}{lcccc}
\hline Ano de 2005 & Freqüência & $\%$ & Média & $t$ Student \\
\hline IAFG março & & & 234,67 & $<0,01^{*}$ \\
Muito fraco/Fraco & 78 & 34,7 & & \\
\hline Regular & 97 & 43,1 & & \\
Bom/Muito bom & 50 & 22,2 & & \\
IAGF dezembro & & & 265,89 & $<0,01^{*}$ \\
Muito fraco/Fraco & 48 & 21,3 & & \\
Regular & 97 & 43,1 & & \\
Bom/Muito bom & 80 & 35,6 & & \\
\hline
\end{tabular}

$* p<0,05$

$\mathrm{Na}$ Tabela 3, observam-se as mudanças ocorridas no IAFG dos idosos, sendo que, em março de 2005, 34,7\% dos idosos apresentavam o IAFG classificado como muito fraco ou fraco; já em dezembro, diminuiu para $21,3 \%$ os idosos classificados nessa categoria. Ainda na mesma tabela, destaca-se a melhora de 31,22 pontos no IAFG, mostrando melhoria nesse índice.

$\mathrm{Na}$ Tabela 4, pode-se identificar a comparação do IAFG dos idosos em relação aos estratos etários (60-69; 70-79; e mais de 80 anos) durante o ano de 2005.

Foi verificada diferença em relação ao IAFG de acordo com a faixa etária dos idosos participantes. Conforme se observa na tabela 4 , em março os idosos com mais de 80 anos apresentaram a melhor média de IAFG, diferindo estatisticamente 
dos demais grupos. Já, na avaliação de dezembro, a média do IAFG do grupo de 60-69 anos foi estatisticamente igual aos demais grupos, sendo que a diferença foi identificada entre os grupos de 70-79 anos e aqueles com mais de 80 anos, tendo este último, a maior média de IAFG.

$\mathrm{Na}$ Tabela 5, percebe-se a interferência que o tempo de prática da atividade física exerce sobre o IAFG dos idosos participantes do programa de atividades físicas.

Tabela 4. Médias do IAFG entre as diferentes faixas etárias entre os meses de março e dezembro de 2005.

\begin{tabular}{lcc}
\hline Faixa etária & IAFG - Março & IAFG - Dezembro \\
\hline $60-69$ & 241,90 & 276,22 \\
$70-79$ & 217,00 & $247,49^{* *}$ \\
mais de 80 & $295,45^{*}$ & 297,50 \\
\hline
\end{tabular}

*difere estatisticamente dos demais grupos por meio do teste da ANOVA $(p<0,05)$.

** difere estatisticamente do grupo com mais de 80 anos por meio do teste da ANOVA $(p<0,05)$.

Tabela 5. Médias do IAFG conforme o tempo de prática dos idosos entre março e dezembro de $2005(n=225)$.

\begin{tabular}{lcc}
\hline Tempo de prática & IAFG - Março & IAFG - Dezembro \\
\hline Até 3 anos de prática & 200,68 & 239,77 \\
3 a 10 anos de prática & 227,57 & 256,63 \\
$\begin{array}{l}\text { Acima de } 10 \text { anos de } \\
\text { prática }\end{array}$ & $260,48^{*}$ & $289,17^{*}$ \\
\hline
\end{tabular}

*Difere estatisticamente dos demais grupos por meio do teste da ANOVA $(p<0,05)$

Na Tabela 5, identifica-se que, em ambas as avaliações (março e dezembro), o grupo de idosos praticantes de atividades físicas, que está inserido há mais tempo no programa, apresentou melhor média de IAFG e diferiram estatisticamente dos demais grupos.

\section{DISCUSSÃO}

O presente estudo objetivou identificar as mudanças do IAFG, no decorrer de 10 meses, de 225 idosos praticantes de atividades físicas, cuja média de idade é de 69,26 anos (DP=5,685).

Os resultados demonstram que houve melhora do IAFG dos idosos participantes no decorrer de 10 meses. Este fato demonstra que, mesmo os idosos envelhecendo, ainda é possível ganhos na aptidão física das variáveis analisadas, facilitando o desenvolvimento das atividades da vida diária destes. Outros estudos realizados com idosos praticantes de hidroginástica ${ }^{9}$ e idosos praticantes de outras modalidades de atividades físicas ${ }^{10,11}$ identificaram resultados idênticos.
A Tabela 3 apresentou diferenças na distribuição de frequência dos idosos na classificação dentro das categorias do IAFG, no decorrer de 10 meses. Pode se observar que a quantidade de pessoas classificadas em "muito fraco" ou "fraco", na avaliação de março de 2005, diminuiu na avaliação de dezembro de 2005.

Da mesma forma, a quantidade de idosos classificados na categoria "bom" ou "muito bom", aumentou na avaliação de dezembro de 2005. Os idosos apresentaram melhoras nas capacidades físicas avaliadas, o que permite sugerir que aqueles classificados como "muito fraco" ou "fraco" passaram a ser classificados como "regular", e os que estavam na faixa "regular", melhoraram para "bom" e "muito bom".

Quando comparados em relação ao estrato etário, foi possível identificar que os idosos com mais idade (acima de 80 anos) apresentaram melhor IAFG, na avaliação realizada no mês de março, o que contrapõe os achados da literatura ${ }^{12}$. $\mathrm{Na}$ avaliação realizada no mês de dezembro, o mesmo grupo etário apresentou a melhor média, porém foi estatisticamente igual à média encontrada com o estrato etário de 60-69 anos.

Destaca-se que os resultados identificados refletem a influência da atividade física, pois o grupo etário acima de 80 anos, com as melhores médias do IAFG, representa o grupo que pratica atividade física pelo maior período de tempo.

Observou-se que o grupo que é praticante há mais de 10 anos é composto por aqueles que apresentam o melhor IAFG, nas avaliações realizadas, tanto no mês de março quanto no mês de dezembro. Aponta-se que este resultado pode estar vinculado à assiduidade e ao tempo de inserção no programa.

Ao avaliar os testes que compõem o IAFG, foram verificadas diferenças estatisticamente significantes nas variáveis coordenação e agilidade/ equilíbrio dinâmico $(\mathrm{p}<0,01)$ entre as duas avaliações realizadas (março/dezembro), e a flexibilidade, resistência de força de membros superiores e resistência aeróbia geral não apresentaram mudanças significativas.

Os resultados do presente estudo corroboram os de Nakamura et al. ${ }^{4}$, que evidenciaram melhora das variáveis coordenação e agilidade/equilíbrio dinâmico, depois de 12 semanas de inserção em atividades físicas, com o grupo que praticou atividades três vezes por semana e também, com o estudo de Sebastião et al..$^{13}$, que por meio da bateria de testes AAHPERD ${ }^{7}$, avaliaram a interferência de quatro meses de dança na capacidade funcional de 
21 idosas (61 $\pm 7,12$ anos) e identificaram mudanças positivas em duas capacidades físicas, a resistência de força e a coordenação motora.

Pauli et al. ${ }^{11}$ também identificaram mudanças significantes na coordenação, flexibilidade e agilidade/equilíbrio dinâmico de idosas, após 12 anos de participação em um programa supervisionado. Os estudos de Carral \& Pérez ${ }^{14}$, Cyarto et al. ${ }^{15} \mathrm{e}$ Cornillon et al. ${ }^{16}$, também verificaram melhoria da agilidade/equilíbrio dinâmico em idosos praticantes de atividades físicas.

Nos resultados obtidos com o presente estudo, identificou-se que não houve melhora significante na flexibilidade, na resistência de força de membros superiores, e na resistência aeróbia geral dos idosos. Porém, cabe destacar a importância da manutenção destas variáveis no período de 10 meses, o que já pode ser considerado um aspecto positivo diante dos efeitos inerentes ao envelhecimento. Segundo Sebastião et al. ${ }^{13}$, por meio da atividade física é possível obter melhora e manutenção na aptidão funcional dos idosos.

Matsudo et al. ${ }^{17}$ verificaram que a capacidade física de mulheres da sexta à oitava década da vida, participantes de um programa regular de atividade física, permaneceram estáveis. No presente estudo, também se mantiveram estáveis as variáveis da flexibilidade, resistência de força de membros superiores e resistência aeróbia geral, permitindo inferir que a prática de atividades físicas é importante para a manutenção destas capacidades dentro de um nível adequado para a independência.

A manutenção da resistência de força, após treinamento de 6 meses, com ginástica localizada em idosas, também foi identificado por Rocha et al. ${ }^{18}$. Michelin et al. ${ }^{19}$ não encontraram mudanças na resistência de força dos membros superiores, quando avaliaram idosos depois de 9 meses de treinamento. Os autores sugeriram relação desses resultados com as perdas de forças inerentes ao processo de envelhecimento.

Diante dos resultados identificados, sugere-se que para manutenção ou evolução das capacidades físicas depende de fatores, como: enfoque de trabalho das aulas oferecidas, heterogeneidade das turmas (diferentes faixas etárias, tempo de inserção no programa e capacidade funcional), assiduidade $\mathrm{e}$ nível de esforço dos idosos para obterem melhoras. Neste sentido, é importante salientar a dificuldade em aumentar a carga (peso dos halteres) devido à resistência (medo) dos idosos em levantar pesos, pelos mitos adquiridos ao longo de sua existência, sendo necessário um trabalho árduo de convencimento e explicações dos ganhos fisiológicos.
O trabalho de resistência de força depende da combinação de variáveis, como o número de repetições, séries, sobrecarga, sequência e intervalos entre as séries e os exercícios, mas ainda suscita dúvidas e merece investigações ${ }^{20}$. Diante disso, infere-se que o fato de não ter sido identificada diferença significante na variável resistência de força de membros superiores, entre as duas avaliações, pode estar relacionado ao fato de não ter havido um controle rígido em relação a todos os aspectos que compõem o trabalho de força.

A não evolução da resistência aeróbia geral, por exemplo, pode estar atrelada ao pouco enfoque dado a esta capacidade nas aulas, justificado pelo pequeno espaço físico e tempo disponível para cada aula (50 minutos).

A manutenção da flexibilidade pode estar relacionada a falhas no estímulo e na realização dos exercícios pelos idosos, pois com a presença da "dor" provocada com o treinamento da flexibilidade, preferem trabalhar com menor amplitude ou não realizar a atividade proposta, justificado pela presença de doenças ou problemas articulares.

Diante dos resultados, destaca-se a importância do desenvolvimento de campanhas que permitam a conscientização dos idosos em relação à sua participação e assiduidade no programa em que estão inseridos. Sugere-se, também, a realização de novos estudos, especialmente, com a inclusão de um grupo controle, o que permitiria avaliar a interferência da atividade física com maior precisão.

\section{CONCLUSÃO}

O presente estudo identificou melhora no IAFG de idosos, no decorrer dos 10 meses de participação em um programa de atividades físicas, sendo que a agilidade/ equilíbrio dinâmico e coordenação foram as variáveis que tiveram alterações significantes e positivas.

As variáveis resistência de força de membros superiores, resistência aeróbia geral e flexibilidade não tiveram alterações significantes, porém evidencia-se a importância na manutenção destas. Podemos constatar que a atividade física foi um dos fatores que colaborou para as melhoras identificadas, especialmente, nos idosos acima de 80 anos e aqueles praticantes de atividades físicas há mais de 10 anos.

Assim, a prática de atividades físicas por idosos pode ser um importante fator para a manutenção da aptidão funcional no decorrer do processo de envelhecimento. Percebe-se, também, a necessidade de campanhas que fomentem a conscientização dos idosos em relação à importância de sua participação 
nos programas de atividades físicas, permitindo obtenção de resultados satisfatórios.

\section{REFERÊNCIAS BIBLIOGRFÁFICAS}

1. Fiedler MM, Peres KG. Capacidade funcional e fatores associados em idosos do Sul do Brasil: um estudo de base populacional. Cad Saúde Pública 2008;24(2):409-415.

2. Gobbi S. Atividade física para pessoas idosas e recomendações da Organização Mundial de Saúde de 1996. Rev Bras Ativ Física Saúde 1997;2(2):41-49.

3. Bocalini DS, Santos L dos, Serra AJ. Physical exercise improves the functional capacity and quality of life in patients with heart failure. Clinics 2008;63:437-42.

4. Nakamura Y, Tanaka K, Yabushita N, Sakai T, Shigematsu R. Effects of exercise frequency on functional fitness in older adult women. Arch Gerontol Geriatr 2007;44(2): 163-173.

5. Thomas J, Nelson J. Métodos de Pesquisa em Atividade Física. Porto Alegre: Artmed, 2002

6. Clark BA. Tests for fitness in older adults: AAHPERD Fitness Task Force. Am J Health Educ 1989;60(3):66-71.

7. Osness WH, Andrian M, Clark BA, et al. Functional Fitness Assessment for Adults Over 60 Years. The American Alliance For Health, Physical Education, Recreation and Dance. Association for research, administration, professional councils, and societies. Council on aging and adult development.Association Drive. Reston; 1990.

8. Zago A, Gobbi S. Valores normativos da aptidão funcional de mulheres de 60 a 70 anos. Rev Bras Ciênc Mov 2003;9(1):28-36.

9. Alves RV, Mota J, Costa M da C, Alves JGB. Aptidão física relacionada à saúde de idosos: influência da hidroginástica. Rev Bras Med Esporte 2004;10(1): 31-37.

10. Sierpowska Á, Ciechanowicz I, Wasilewska GC. Functional Fitness Assessment Among Elderly Women (60+) Participanting in Yoga or Swimming Exercises. Stud Physl Cult Tourism 2006;13(Suppl):81-83.

11. Pauli, JR; Souza, LS, Zago, AS, Gobbi, S. Influência de 12 anos de prática de atividade física regular em programa supervisionado para idosos. Rev Bras Cineantropom Desempenho Hum 2009; 11(3):255-260.

12. Lima-Costa MF, Barreto SM, Giatti LG. Condições de saúde, capacidade funcional, uso de serviços de saúde e gastos com medicamentos da população idosa brasileira: um estudo descritivo baseado na Pesquisa Nacional por Amostra de Domicílios. Cad Saúde Pública 2003;19(3):735-743.
13. Sebastião E, Hamanaka AYY, Gobbi LTB, Gobbi S. Efeitos da Prática Regular de Dança na Capacidade Funcional de Mulheres acima de 50 anos. Rev Educ Fís 2008; 19(2):205-214.

14. Carral JMC, Pérez CA. Effects of High-Intensity Combined Training on Women over 65. Gerontology 2007;53(1):340-346.

15. Cyarto EV, Brown WJ, Marshall AL, Trost SG. Comparison of the Effects of a Home-Based and Group-Based Resistance Training Program on Functional Ability in Older Adults. Am J Health Promot 2008;23(1):13-17.

16. Cornillon E, Blanchon MA, Ramboatsisetraina P, Braize C, Beauchet $\mathrm{O}$, et al. Effectiveness of falls prevetions strategies for elderly subjects who live in the community with performance assessment of physical activities (before-after). Ann Readapt Med Phys 2002;45(9):493-504.

17. Matsudo SM, Matsudo VKR, Neto TL de B, Araújo TL de. Evolução do perfil neuromotor e capacidade funcional de mulheres fisicamente ativas de acordo com a idade cronológica. Rev Bras Med Esporte 2003;9(6):365-376.

18. Rocha AC, Fernandes MC, Dubas JP, Guedes Júnior DP. Comparative analysis of muscular force between elderly women practitioners pf weight training, institutionalized and located gymnastics. Fit Perf J 2009;8(1):16-20.

19. Michelin E, Coelho CF, Birini RC. Efeitos de um mês de destreinamento sobre a Aptidão Física Relacionada à Saúde em Programam de Mudança de Estilo de Vida. Rev Bras Med Esporte 2008;14(3):192-196.

20. Silva NLD, Farinatti PTV. Influência de variáveis do treinamento contra-resistência sobre a força muscular de idosos: uma revisão sistemática com ênfase nas relações dose-resposta. Rev Bras Med Esporte 2007;13(1):60-66.

\footnotetext{
Endereço para correspondência

Natália Cristina Santos Cipriani

Rua Lauro Linhares, nº 1830, Bloco Venezuela, Apto 104. Bairro - Trindade.

CEP: 88036-002 - Florianópolis, SC. Brasil

E-mail: nataliacipriani@gmail.com
} 